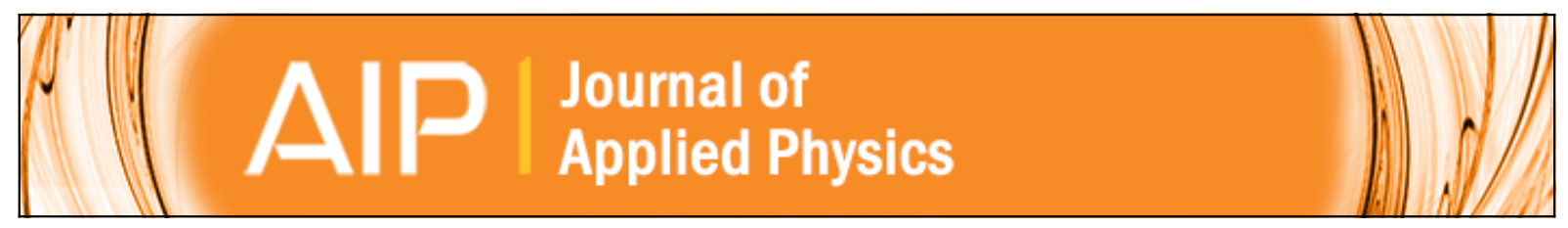

\title{
A model for the dependence of the electrical conductance with the applied stress in insulating-conducting composites
}

R. Martín Negri, Silvio D. Rodriguez, Delia L. Bernik, Fernando V. Molina, Ana Pilosof, and Oscar Perez

Citation: Journal of Applied Physics 107, 113703 (2010); doi: 10.1063/1.3410799

View online: http://dx.doi.org/10.1063/1.3410799

View Table of Contents: http://scitation.aip.org/content/aip/journal/jap/107/11?ver=pdfcov

Published by the AIP Publishing

\section{Articles you may be interested in}

Pressure-dependent electrical conductivity of freestanding three-dimensional carbon nanotube network Appl. Phys. Lett. 102, 183117 (2013); 10.1063/1.4804385

Electrical conductivity of platinum-implanted polymethylmethacrylate nanocomposite

J. Appl. Phys. 110, 114905 (2011); 10.1063/1.3668096

Magneto-piezoresistance in elastomagnetic composites

J. Appl. Phys. 110, 063903 (2011); 10.1063/1.3634120

Apparent dependence of conductivity of a conducting polymer on an electric field in a field effect transistor configuration

J. Appl. Phys. 92, 6033 (2002); 10.1063/1.1511291

Conduction mechanisms in some graphite-polymer composites: Effects of temperature and hydrostatic pressure J. Appl. Phys. 83, 1410 (1998); 10.1063/1.366904

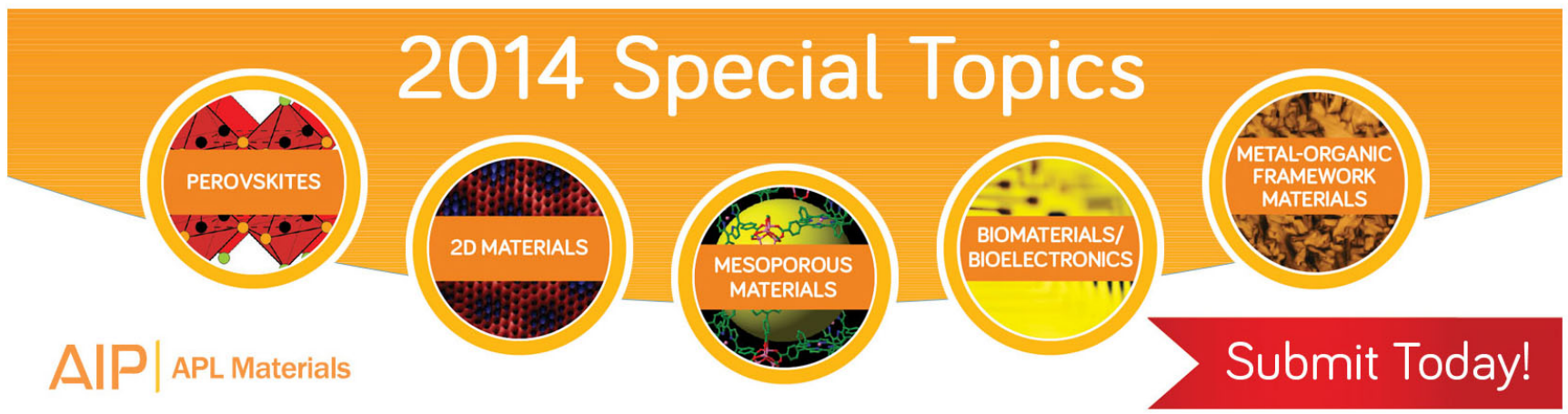




\title{
A model for the dependence of the electrical conductance with the applied stress in insulating-conducting composites
}

\author{
R. Martín Negri, ${ }^{1, a)}$ Silvio D. Rodriguez, ${ }^{1}$ Delia L. Bernik, ${ }^{1}$ Fernando V. Molina, ${ }^{1}$ \\ Ana Pilosof, ${ }^{2}$ and Oscar Perez ${ }^{2}$ \\ ${ }^{1}$ Departamento de Química Inorgánica, Instituto de Química Física de Materiales, Ambiente y Energía \\ (INQUIMAE), Analítica y Química Física, Facultad de Ciencias Exactas y Naturales, Universidad de Buenos \\ Aires, C1428EGA, Buenos Aires, Argentina \\ ${ }^{2}$ Departamento de Industrias, Facultad de Ciencias Exactas y Naturales, Universidad de Buenos Aires, \\ C1428EGA, Buenos Aires, Argentina
}

(Received 16 November 2009; accepted 27 March 2010; published online 2 June 2010)

\begin{abstract}
A model for the dependence of the electrical conductance, $G$, with the strain induced by external mechanical stress in conducting particles-polymer composites is presented. The model assumes that the percolation probability between neighboring particles must depart from a scale-invariant behavior but saturate at moderated-high strains, reaching percolation path's saturation, with sigmoid dependence. This dependence is obtained by proposing a dynamic picture where contacts or bonds between neighboring particles are created but also destructed when a stress is applied and relatively moderated or high strains, $\varepsilon$, are produced in the composite. The electrical conductance of prepared graphite-polydimethylsiloxane composites were measured as function of the applied pressure and fitted by the presented model. The elastic response to the uniaxial compression was studied using a texture analyzer. The possibility of nonuniversal effects in the conduction critical exponent, $t$, was taken into account. It is concluded that the saturation of the response in the $G$ versus $\varepsilon$ plots cannot be assigned to nonuniversal behavior of the exponent $t$, or to saturation of the elastic response. On the other hand, the presented model accounts for all the main experimental features observed in these systems and for previously reported data of elastomer composites. The simulated behavior of the piezoresistivity coefficient is also in qualitative agreement with previous reports. (C) 2010 American Institute of Physics. [doi:10.1063/1.3410799]
\end{abstract}

\section{INTRODUCTION}

One of the most interesting issues in theoretical and experimental studies of disordered insulator-conductor composites is the effect on the transport properties of external perturbations, like electric and magnetic fields, mechanical stress, or molecular adsorption. ${ }^{1-12}$ We are focusing in the present work on composites of conducting particles embedded in an insulating elastomer matrix. The matrix is compressed by the action of an externally applied uniaxial pressure keeping constant the amount of conducting particles, a process that increases the electrical conductance of the composite, which can be used for obtaining a pressure sensor. These systems are usually considered as isotropic percolating networks where the dependence of transport properties, like the dc-electrical conductance, $G$, are expressed as functions of the percolation probability between neighboring particles. ${ }^{13-27}$ Many experimental works have been reported concerning the effect of mechanical stress on $G$ (Refs. 9, 19, and 28-39) and the ac-impedance of composites, ${ }^{38-42}$ but the systematic theoretical investigation of the dependence of $G$ with the external applied stress has been initiated in a few relevant works in recent years. ${ }^{13-17,20,21,24}$

\footnotetext{
a) Author to whom correspondence should be addressed. Present address: Ciudad Universitaria, Pabellón II. INQUIMAE. C1428EGA, Buenos Aires, Argentina. Electronic mail: rmn@qi.fcen.uba.ar. Tel.: xx54-11-4576-3358. FAX: xx54-11-4576-3341.
}

The main experimental features commonly observed in pressure sensors based on elastomer composites are high sensitivity at low applied stress, currently with exponential rise of $G$ as function of the applied pressure, followed by saturation. ${ }^{29,35,36,39}$ The aim of this work is to contribute to the discussion about the dependence of $G$ with the external applied stress (and the resulting strain) by introducing a model which accounts for these features. Polydimethysiloxane (PDMS)/graphite composites were prepared and $G$ versus strain plots obtained, illustrating these characteristics. Elastic measurements were performed also in order to determine the elastic response to the external uniaxial pressure. Experimental data were fitted by the introduced model, which allows including nonuniversal behavior of the critical exponent for electrical conduction in percolation networks.

\section{VISCOELASTICIY AND PIEZORESISTIVITY IN NONUNIVERSAL CONDUCTION}

\section{A. Elastic response}

We focus on films of an elastomer-conducting composite having a thickness $L$ of about $1 \mathrm{~mm}$, which decreases with the applied pressure, and an area $A\left(\sim 1 \mathrm{~cm}^{2}\right)$ which is kept approximately constant during compression. The uniaxial pressure is applied parallel to the electrical current direction, but there are two ways of performing compression. The first one is compressing the film at a constant speed (e.g., 0.1 $\mathrm{mm} / \mathrm{s}$ ) from an initial value, $L_{i}$, up to reaching a defined final 
thickness, typically $70 \%-90 \%$ of $L_{i}$. In this case, the pressure on the film can be measured in real time during the compression using a texture analyzer device; the Young's modulus of the film, $E$, is obtained from these experiments. In the second case, the film is placed on a balance and a constant pressure is applied, comprising the film up to a final thickness. In both cases, the electrical resistance of the films can be recorded as function of the applied pressure.

The longitudinal strain, $\varepsilon$, parallel to the direction of the applied pressure and the electrical current, is defined in differential terms by

$$
d \varepsilon=\frac{d L}{L}
$$

where $L$ is the thickness of the film, $L \leq L_{i}$, with $L_{i}$ the initial thickness (at $\varepsilon=0$ ), defined during the preparation of the film. The relationship between $\varepsilon$ and the applied pressure, $P$, is assumed to follow the Young's law:

$$
d \varepsilon=-\frac{d P}{E},
$$

where $E$ is the Young's modulus of the film. Integration of these expressions renders to

$$
\begin{aligned}
& L(\varepsilon)=L_{i} \exp (\varepsilon), \\
& \varepsilon=-\frac{P}{E},
\end{aligned}
$$

with $\varepsilon \leq 0$ and $P \geq 0$. Note that $\varepsilon$ is negative because only compressions but not elongations are considered.

The above equalities explicitly indicate that the actual master variable is $\varepsilon(\equiv-P / E)$ instead of $P$, then all the relevant physical properties are expressed as functions of $\varepsilon$. We distinguish in the present work between low strains $(|\varepsilon|$ $\left.\sim 10^{-4}-10^{-3}\right)$ and moderated strains $(|\varepsilon| \sim 0.01-0.3)$. Very good fits of $L$ versus $\varepsilon$ or $P$ using Eqs. (3) and (4) in the range $|\varepsilon| \leq 0.3$ were obtained using the texture analyzer for PDMS-graphite films, that is, the observed variation in $L$ with $\varepsilon$ was exponential with no saturation in that range. In some cases, excellent fits of $L$ versus $\varepsilon$ were obtaining by linearization of Eq. (3): $L(\varepsilon) \cong L_{i}(1-\varepsilon)$. These results are described in Sec. VI B.

\section{B. Piezoresistivity}

The macroscopic electrical conductance, $G,(\equiv 1 / R$, where $R$ is the electrical resistance of the films at a fixed voltage $V$ ) is related, at each applied stress or strain field, to the dc electrical conductance, $\sigma$, and to $L$

$$
G(\varepsilon)=\frac{1}{R}=\frac{A \sigma(\varepsilon)}{L(\varepsilon)} .
$$

In the range of applied strains considered in the present work $\left(|\varepsilon| \sim 10^{-2}-10^{-1}\right)$, a strong dependence of $\sigma$ with $\varepsilon$ is expected, thus it varies during the compression. Hence, $G(\varepsilon)$ is the magnitude easily accessible by experiments and the central objective is to obtain a model for its dependence with $\varepsilon$, which comes from two contributions, $L$ and $\sigma$, in Eq. (5) (the area $A$ is considered constant in the present work). The ex- plicit dependence with $L$ is the trivial one because the electric field on the film, $V / L$, increases when compressing at a fixed voltage and this is the origin of the term $1 / L$ in Eq. (5). Although this contribution will be considered in the formalism, we advance that numerically is not relevant at all in the range of strains considered.

The dependence of $\sigma$ with $\varepsilon$ is usually introduced through the so-called piezoresistive factor, $\Gamma_{\|}(\varepsilon)$,

$$
\Gamma_{\|}(\varepsilon) \equiv-\frac{d \ln \sigma}{d \varepsilon}
$$

where the subindex $\|$ stands for the fact that the considered stress is parallel to the electrical current. Integration of Eqs. (5) and (6) renders to

$$
\frac{G(\varepsilon)}{G_{i}}=\exp \left(-\varepsilon-\int_{\varepsilon}^{0} \Gamma_{\|}(\varepsilon) d \varepsilon\right),
$$

where $G_{i} \equiv G(\varepsilon=0)$. Although only experimental results concerning with $G / G_{i}$ are shown, the theoretical expressions not only for $G / G_{i}$ but also for $\Gamma_{\|}$are deduced and presented in Sec. II C, because $\Gamma_{\|}$is a relevant property of the system that can be, eventually, determined by experiments.

Equality (7) must be modified for those cases where the area $A$ changes during compression, obtaining $G(\varepsilon) / G_{i}$ $=\exp \left[-\left(1+2 \nu_{P}\right) \varepsilon-\int_{\varepsilon}^{0} \Gamma_{\|}(\varepsilon) d \varepsilon\right]$, where $\nu_{P}$ is the Poisson's modulus, as correctly indicated by Carmona et al. ${ }^{39}$

\section{Conduction criterions and general relationships}

The connectivity of particles in composites is described in terms of the percolation theory, although several aspects are far from being completely understood as remarked recently by Balberg. ${ }^{14}$ Following the discussion made in Ref. 14, composites are usually described as a site percolating network where a lattice of sites is partially occupied by conducting particles. If two nearest neighboring sites are both occupied, then they are locally connected; a group of connected sites is called a cluster. The probability of site occupation is denoted by the symbol $p$ in this model. There is connection between extremes of the lattices above a critical value, $p_{c}$, and then conduction appears provided by a tortuous path while current flows through some parts of the percolation cluster referred as the backbone of the percolation cluster. This lattice picture can be coupled to different physical models of conductivity between particles, which is usually defined as the conductivity criterion. For instance, the lattice model can be coupled to the Scher and Zallen model where the conducting particles are considered as spheres which occupy the lattice sites and where direct geometrical (physical) contact between the spheres is assumed. The effect of external stress has not been discussed in the frame of this model, as far as we know.

In addition to the above model, a more indirect mechanism of conduction is also present in real samples, quantum tunneling between particles. According to Balberg, "quantum tunneling mechanism is the only carrier transport mechanism between particles that do not touch geometrically." 14 Therefore, tunneling can be the dominant mechanism when the 
concentration of the conducting particles is relatively low, that is when the system is close to the critical point for conduction. Tunneling is not described as a site percolation network but as a bond percolation: particles are placed at sites but there is a distribution of conducting bonds between them as function of the distance between particles. Balberg showed the coexistence of the site percolation connectivity (tortuous paths) and the tunneling connectivity (all particles, in principle, are electrically connected). The percolation threshold for this tunneling-percolation mechanism is determined then by the particle concentration necessary for that network to form. This picture, the coexistence of a lattice of sites together with a subnetwork of "tunneling bonds" seems to be at present the more developed description of the socalled tunneling-percolation model (TPM). ${ }^{13,14,16,17,20,21,24,27}$

In practice, there is no simple manner to distinguish between direct contact and tunneling. Hence, both possibilities are kept, as already indicated by Kchit and Bossis. ${ }^{1}$ We assume that the net passage of an electron from one particle to another neighbor can be due to both mechanisms (contact or tunneling), with a global probability $p$ whose critical value for connectivity between extremes is referred as $p_{c}$. In the present work, we focalize on the conductance changes observed as function of the strain while keeping constant the amount of particles. In this picture, $p$ changes with the strain because bonds and/or contacts are created-destructed at the relatively large strains considered here. The possibility of a dynamic process were contact-bonds are not only created but also destructed has not been considered in the few reported works concerning with stress effects, because low concentrations and strains were considered, but it has been mentioned in Ref. 21. This is the basis of the model presented in Sec. III, referred as the percolation saturated model (PSM), thought to be applied in the range of large particle concentrations and strains. The critical value $p_{c}$ is reasonably considered as dependent of the chemical nature of the composite but not on the strain field.

Before presenting the PSM, general relationships for $\sigma$, $\Gamma_{\|}$, and $G$ as functions of $\left(p-p_{c}\right)$ are shown in this section. These expressions are written in terms of the so-called conductance critical exponent function, $t(\varepsilon)$ : it is assumed that the amount of particles is large enough to assure nonzero conductance, even at $\varepsilon=0$. Consequently, the probability $p$ must be always larger than $p_{c}$, in the model to be developed $\left(p>p_{c}\right)$

$$
t(\varepsilon) \equiv \frac{d \ln \sigma}{d \ln \left(p-p_{c}\right)} \quad \text { for } \quad p>p_{c} .
$$

The origin of the definition Eq. (8) comes from the standard percolation theory (SPT) (Refs. 43 and 44) where $\sigma$ is predicted to obey the scale-invariance property which establish that the relative differential changes of $\sigma$ must be proportional to the relative differential changes of $\left(p-p_{c}\right)$, that is: $\mathrm{d} \sigma / \sigma=t_{0} \mathrm{~d}\left(p-p_{c}\right) /\left(p-p_{c}\right)$. In that case, the proportional factor $t_{0}$ is predicted to be a universal constant $\left[t_{0}=2\right.$ in threedimensional (3D) materials], then $\sigma$ should follow a power's law dependence with $\left(p-p_{c}\right): \sigma \propto\left(p-p_{c}\right)^{t_{0}}$ and $t_{0}$ has the meaning of the fractal dimension for conduction. The universal behavior describes well the experimental results in many cases when the percolation probability is changed using samples with different amount of conducting particles and particularly in materials which are prepared with concentrations close to the conductivity threshold ( $p$ close to $p_{c}$ ). Nevertheless, nonuniversal behavior has been experimentally observed and theoretically predicted in the last years. ${ }^{20,21,30,43-46}$

The above definition [Eq. (8)] holds for the whole range of percolation probabilities, that is, even for $p$ not close to $p_{c}$. For those cases, Eq. (8) represents the local definition of the function $t(\varepsilon)$, which is not a universal constant but expected to be dependent on the specific composite material (chemical nature and morphology of the conducting particles and polymer), the concentration of the conducting particles and also of the strain in the range of moderated or large strains $(|\varepsilon|$ $\left.\sim 10^{-2}-0.03\right)$. In that range, scale invariance is not expected to hold, that is $t(\varepsilon)$ is not expected to be a constant but a function of the strain field. For this reason, Eq. (8) is a derivative expression while integrated relationships, like $\sigma$ $=\sigma_{o}\left(p-p_{c}\right)^{t}$, are not longer valid in the considered range of conductance-strain far from the percolation threshold. Variations in $t$ with $\varepsilon$ have been experimentally presented by Johner et al., ${ }^{17}$ although for relatively small values of $\varepsilon(|\varepsilon|$ $\sim 10^{-4}$ ) and at the vicinity of the percolation threshold. Therefore, we present in this section the expressions of $\sigma, \Gamma_{\|}$, and $G$ in terms of $t(\varepsilon)$ and $\left(p-p_{c}\right)(\varepsilon)$ without assuming any functional dependence of $t(\varepsilon)$ and $\left(p-p_{c}\right)(\varepsilon)$ with $\varepsilon$, which must be valid in the whole range of possible strains.

Integration of Eqs. (7) and (8) is performed under the following boundary conditions:

$$
\begin{aligned}
& p(\varepsilon=-\infty)=1, \\
& p(\varepsilon=0) \equiv p_{i}, \\
& \sigma(\varepsilon=0) \equiv \sigma_{i}, \\
& G(\varepsilon=0) \equiv G_{i}, \\
& t(\varepsilon=0) \equiv t_{i} .
\end{aligned}
$$

The boundary condition Eq. (9a) is a mathematically helpful idealization which simplify calculations; it just indicates that the probability $p$ reaches a maximum (assumed equal to one) at large strains.

Integration of Eqs. (6)-(8) with the above boundary conditions renders to

$$
\begin{aligned}
& \Gamma_{\|}(\varepsilon)=-t(\varepsilon) \frac{d \ln \left[p(\varepsilon)-p_{c}\right]}{d \varepsilon}, \\
& \frac{\sigma(\varepsilon)}{\sigma_{i}}=\frac{\left[p(\varepsilon)-p_{c}\right]^{t(\varepsilon)}}{\left(p_{i}-p_{c}\right)^{t_{i}}} \exp \left\{\int_{\varepsilon}^{0}\left(\frac{d t}{d \varepsilon}\right) \ln \left[p(\varepsilon)-p_{c}\right] d \varepsilon\right\},
\end{aligned}
$$




$$
\begin{aligned}
\frac{G(\varepsilon)}{G_{i}}= & \underbrace{\exp (-\varepsilon)}_{G_{1}(\varepsilon)} \underbrace{\frac{\left[p(\varepsilon)-p_{c}\right]^{t(\varepsilon)}}{\left(p_{i}-p_{c}\right)^{t_{i}}}}_{G_{2}(\varepsilon)} \\
& \underbrace{\times \exp \left\{\int_{\varepsilon}^{0}\left(\frac{d t}{d \varepsilon}\right) \ln \left[p(\varepsilon)-p_{c}\right] d \varepsilon\right\}}_{G_{3}(\varepsilon)} .
\end{aligned}
$$

Note that $t_{i}$ can be different of $t_{0}$, the universal value predicted by the SPT $\left(t_{0}=2\right.$ in 3D composites). It is assumed also that the amount of particles in the composite is large enough to satisfy the condition $p>p_{c}$ in the whole range of applied pressures, even when the film is not compressed $(\varepsilon$ $=0$ ). The conductance at the critical threshold, $\sigma_{c}$, is assumed equal to zero. These conditions are assumed here for simplicity, although can be relaxed and the formalism eventually extended.

$G(\varepsilon) / G_{i}$ is given in Eq. (12) by a product of three functions, $G_{1}(\varepsilon) G_{2}(\varepsilon) G_{3}(\varepsilon)$. The first one, $G_{1}(\varepsilon)$, represents the trivial contribution because of compressing the film at a fixed voltage, with $G_{1}(\varepsilon)=1 / L=\exp (-\varepsilon)$ if the area $A$ is constant, as assumed here [otherwise $G_{1}(\varepsilon)=\exp \left[-\left(2 \nu_{p}+1\right) \varepsilon\right]$, where $\nu_{p}$ is the Poisson's modulus $\left.{ }^{39}\right]$. The second terms, $G_{2}(\varepsilon)$ keeps the formal expression of the power-law dependence between $\sigma$ and $\left(p-p_{c}\right)$, but modified in order to incorporate nonuniversality effects.

The last product, $G_{3}(\varepsilon) \equiv \exp \left\{\int_{\varepsilon}^{0}(d t / d \varepsilon) \ln \left[p(\varepsilon)-p_{c}\right] d \varepsilon\right.$, is exclusively due to nonuniversality effects.

Hence, the above expressions are obtained without any lack of generality. In Secs. III and IV, we propose and use models for the dependences of $t(\varepsilon)$ and $\left(p-p_{c}\right)(\varepsilon)$ with $\varepsilon$, whose predictions are in agreement with the experimental features observed in pressure sensors.

\section{MODELING THE RELATIVE PERCOLATION PROBABILITY, $p(\varepsilon)$}

Expressions (10)-(12) are formally given in terms of $t(\varepsilon)$ and $p(\varepsilon)-p_{c}$. The dependence of $t(\varepsilon)$ is discussed in Sec. IV, while models for the dependence of $p(\varepsilon)-p_{c}$ with $\varepsilon$ are introduced here.

The relevant magnitude for the consequent analysis is $\left(p-p_{c}\right)$ (but not the individual values of $p$ and $p_{c}$ ) which is expected to be dependent of the strain, $\varepsilon$. In principle, all the relevant magnitudes can be modified by changing the strain field $(\varepsilon)$ at a fixed weight fraction of conducting particles, $X$, or vice versa by changing $X$ at fixed $\varepsilon$

$$
d\left(p-p_{c}\right)=\left(\frac{\partial\left(p-p_{c}\right)}{\partial \varepsilon}\right)_{X} d \varepsilon+\left(\frac{\partial\left(p-p_{c}\right)}{\partial X}\right)_{\varepsilon} d X
$$

The more current manner to change $p-p_{c}$ is by intersample experiments where $X$ is modified between samples and $\varepsilon$ $=0$ (or constant), thus, $\left(\partial\left(p-p_{c}\right) / \partial X\right)_{\varepsilon}$ is the magnitude actually obtained in those experiments [usually a linear dependence between $p$ and $X$ is obtained at the vicinity of the critical point when keeping $\varepsilon=0$, that is $\left(\partial\left(p-p_{c}\right) / \partial X\right)_{\varepsilon}$ $=$ constant near $\left.p_{c}\right]$. However, in the present work, $\left(p-p_{c}\right)$ changes in a given sample because of the external stress, which modifies the strain field $\varepsilon$ while keeping constant the amount of conducting particles representing intrasample changes. Thus, $\left(\partial\left(p-p_{c}\right) / \partial \varepsilon\right)_{X}$ is the magnitude to be modeled in the present work, representing the dependence of dependence of $\left(p-p_{c}\right)$ as function of the applied stress. It must be noted that changes of $\left(p-p_{c}\right)$ with the applied stress must be taken into account because of the relatively large strains considered here, which represents a central difference with respect to Ref. 17 where very small strains were used and therefore the dependence of $p-p_{c}$ with $\varepsilon$ was reasonably neglected. Hence, in terms of differential equations, the differential strain, $d \varepsilon$ is the origin of the differential changes $d \sigma$ and $d\left(p-p_{c}\right)$.

In practice, not the absolute changes but the relative changes of $p-p_{c}$ are usually considered, that is $1 /\left(p-p_{c}\right)$ $\times\left[\partial\left(p-p_{c}\right) / \partial \varepsilon\right]_{X}=\left\{\partial\left[\operatorname{Ln}\left(p-p_{c}\right)\right] / \partial \varepsilon\right\}_{X}$. This arises in the fact that in critical phenomena the relative changes between the different magnitudes are compared, see Eq. (8) as an example, and where scale-invariant relationships are currently established at the vicinity of the critical point. For instance, if $x$ and $y$ are the variables, they are connected by a scaleinvariance relationship if the relative changes of $y$ are proportional to the relative changes of $x: \Delta y / y \propto \Delta x / x$, which in differential terms are expressed by: $\mathrm{d} y / y=k \mathrm{~d} x / x$, [or: $\mathrm{d}(\ln y)=k \mathrm{~d}(\ln x)]$ where $k$ is a proportionality constant. As a consequence, $y$ follows a power law dependence with $x: y \approx x^{k}$, usually described as a fractal behavior, where $k$ is referred as the critical or fractal exponent. This kind of behavior is predicted to be followed, for instance, by $\sigma$ and $\left(p-p_{c}\right)$ in systems with universal behavior and also between the density and temperature difference, $\left(\mathrm{T}-\mathrm{T}_{\mathrm{c}}\right)$, in gas-phase transitions close to the critical temperature $T_{c}$.

Therefore, the first model to be considered is an scaleinvariant model (referred as SIM) between $p-p_{c}$ and the variable dimension of the system, $L$, which in turn is inverse proportional to the volume fraction of the conducting particles. This model is presented Sec. III A.

\section{A. SIM}

We first discuss the so-called scale-invariant behavior, referred here as SIM, which is actually the current model used for describing a great variety of critical process. It should be clear that SIM does not account at all for the experimental observations, because cannot predict saturation of the film response with $\varepsilon$. Then, after its critical analysis and comparison of predictions with experimental results, we present a model in Sec. III B, referred as PSM, which converge to SIM at low strains but renders predictions that accounts for the experimentally observed saturation. Nevertheless, the description of SIM is necessary for understanding its limitations and the need for developing a more extensive model (PSM).

Considering that in a pressure sensor, the compression of the film (decrease in $L$ ) modifies the percolation probability (increase in $p-p_{c}$ ), then it seems reasonable, in principle, to propose a scale-invariant relationship between $\left(p-p_{c}\right)$ and $L$ (or the volume fraction $\phi$ ) 


$$
\begin{aligned}
\frac{d\left(\left(p-p_{c}\right)\right.}{\left(p-p_{c}\right)}= & -\beta \frac{d L}{L}=-\beta d \varepsilon \\
& \text { with } X=\text { constant } \quad(\mathrm{SIM})
\end{aligned}
$$

or, strictly

$$
\left(\frac{\partial\left(\operatorname{Ln}\left(p-p_{c}\right)\right.}{\partial \varepsilon}\right)_{X}=-\beta \quad(\operatorname{SIM}) .
$$

Provided that $\beta$ is independent of $\varepsilon$, integration of Eq. (15) gives

$$
p(\varepsilon)-p_{c}=\left(p_{i}-p_{c}\right)\left(\frac{L}{L_{i}}\right)^{-\beta} \quad(\mathrm{SIM}) .
$$

In this expression, $\beta$ is a critical exponent that must be positive in order for $p$ to increase when compressing the film. Considering Eqs. (15) and (3), an exponential dependence between $p-p_{c}$ and $\varepsilon$ is obtained with SIM

$$
p(\varepsilon)-p_{c}=\left(p_{i}-p_{c}\right) \exp (-\beta \varepsilon) \quad(\mathrm{SIM}) .
$$

The above expression predicts a continuous growth of $G(\varepsilon)$ with $|\varepsilon|$ when introduced into Eq. (12). For example, the term $G_{2}(\varepsilon)$ is predicted by SIM to be equal to

$$
G_{2}(\varepsilon)=\frac{\left(p_{i}-p_{c}\right)^{t(\varepsilon)}}{\left(p_{i}-p_{c}\right)^{t_{i}}} \exp [-\beta \varepsilon t(\varepsilon)] \quad(\mathrm{SIM})
$$

provided that $\varepsilon \leq 0, \beta>0$, and $t(\varepsilon)>0$.

We show in Sec. IV that $t(\varepsilon)$ has a small variation with $\varepsilon$ and in many cases can be approximated by a constant equal to $t_{i}$, its value at $\varepsilon=0$. Thus, Eq. (18) can be well approximated by $\exp \left(-\beta t_{i} \varepsilon\right)$. Therefore, the main characteristic of SIM is the prediction of a continuous and approximately exponential rise of the sensor's response with the strain, $G(\varepsilon) \approx G_{1}(\varepsilon) G_{2}(\varepsilon) \approx \exp (-\varepsilon) \exp \left(-\beta t_{i} \varepsilon\right)$, having a characteristic stress-strain constant equal to $1 /\left(1+\beta t_{i}\right)$. Beyond the numerical details, it is clear that SIM can be applied to fit experimental data at very low strains only where $G(\varepsilon)$ suddenly increase with $\varepsilon$, but cannot account for the experimental observations in the whole range of strains because does not predict saturation of the sensor's response with $\varepsilon$. Therefore, a more advanced model, which should reduce to SIM at the limit of low strains, is presented in Sec. III B.

\section{B. PSM}

Following the above discussion, it is necessary to present a model which accounts for saturation effects at moderated strains and which must converge to SIM at very low strains. The model developed in the present section assumes that saturation of the sensor's response is reached because the percolation probability reaches saturation in the range of applied pressures. In other words, it is assumed that the system cannot continuously increase $p$; the possibilities for contact-bonds between neighbor particles cannot indefinitely increase with the strain thus the percolation paths cannot be continuously increased even at high strain.

The first aspect to remark is that this kind of behavior implicates that scale-invariance relationship between ( $p$ $-p_{c}$ ) and $L$ or $\phi$ cannot hold because $p-p_{c}$ cannot indefi- nitely follow (scale) $L$ when $\varepsilon$ increases in the range of moderated-high values. The second aspect is to emphasize the statistical nature of the process of creating bonds between particles induced by the applied stress. In SIM, the change in the bond probability, $\mathrm{d} p$, at any strain is always proportional to the existent bonds at that strain: $\mathrm{d}\left(p-p_{c}\right) \sim\left(p-p_{c}\right) \mathrm{d} \varepsilon$; then the number of bonds continuously increase with $|\varepsilon|$ in SIM. The easiest manner of modifying SIM in order to include saturation effects keeping the SIM dependence at low strains is assuming that the probability of contact-bond creation should be proportional to the product of both the number of existent contact-bonds $(p)$ and the number of nonexisting (broken) contact-bonds between particles which is given by $(1-p)$. The competition between the increasing of $p$ and the simultaneous decreasing of $(1-p)$ renders to saturation at moderated-high strains $(p \rightarrow 1 \Rightarrow \mathrm{d} p \rightarrow 0$ : saturation) and to SIM at low stresses $\left(p \rightarrow p_{c} \Rightarrow \mathrm{d} p \sim-\left(p-p_{c}\right) \mathrm{d} \varepsilon\right.$ : exponential increase with $|\varepsilon|$ ). Besides, an inflection in the behavior of $p$ as function of $\varepsilon$ is expected at intermediate strains indicating the change between both regimes. These three aspects: exponential rise at low strains, inflection, and finally saturation are coincident with the experimental features already observed. Interestingly, Johner et al. ${ }^{13,16}$ reported this kind of behavior for the spanning cluster probability (which must be proportional to the conductance) as function of the conducting particles density, for example when simulating a model of continuum segregated particles. ${ }^{13}$

The exact mathematical development of the above concepts requires a differential expression for $p(\varepsilon)$ compatible with the boundary conditions Eqs. (9a)-(9e), achieved by

$$
\begin{aligned}
\frac{1}{\left(p-p_{c}\right)} d\left(p-p_{c}\right)= & -\beta\left(1-\frac{\left(p-p_{c}\right)}{\left(1-p_{c}\right)}\right) d \varepsilon \\
& \text { with } X=\text { constant } \quad(\mathrm{PSM})
\end{aligned}
$$

or

$$
\frac{1}{\left(p-p_{c}\right)}\left(\frac{\partial\left(p-p_{c}\right)}{\partial \varepsilon}\right)_{X}=-\beta\left(1-\frac{\left(p-p_{c}\right)}{\left(1-p_{c}\right)}\right) \quad(\mathrm{PSM})
$$

The PSM model is based on similar hypothesis to those currently postulated in statistical systems where the main relevant parameter is kept constant. In the present case when postulating that $p(\varepsilon)$ cannot indefinitely increase, it is implicitly assumed that the maximum number of contacts is a constant that cannot be changed by the strain field. Thus, the mathematics is analogous, for instance, to that used in grand canonical ensembles where the total number of particles is kept constant, adapted to the boundary conditions of the present problem. In fact, García Molina et al. ${ }^{47}$ already assumed as an empirical hypothesis that the number of present bonds between neighborhoods in a triangular network is governed by a Fermi-Dirac function. In the present context, Eq. (19) is based on the arguments described in this and previous paragraphs, and also on the fact that bonds and sites are indistinguishable, one individual particle (or bond) has the two only possibilities of belonging or not to a fully connected percolation cluster, and that this possibility must vary with the strain $\varepsilon$ under the condition that the number of 
bonds does not increase indefinitely, even at very large strains. Hence, the situation resembles that observed when using the Fermi-Dirac distribution in solid-state physics where the fermions cannot be continuously accumulated at low temperatures in the lowest energy levels because of the Pauli's Principle. Analogously, we assume here that percolation path cannot be "accumulated" even at high strains.

Integration of Eq. (20) with the boundary conditions gives a sigmoid function for $\left(p-p_{c}\right)$

$$
p(\varepsilon)-p_{c}=\frac{\left(1-p_{c}\right)}{\left\{1+\exp \left[\beta\left(\varepsilon-\mu_{0}\right)\right]\right\}},
$$

where $\beta$ and $\mu_{0}$ are constants. The presence of the constant factor $\left(1-p_{c}\right)$ in Eqs. (20) and (21) is compatible with one of the boundary conditions already mentioned: $p \rightarrow 1$ when $\varepsilon$ $\rightarrow-\infty$ [Eq. (9a)]. The introduction of $\mu_{0}$ is related to the boundary condition $p(\varepsilon=0)=p_{i}$ [Eq. (9b)]

$$
\exp \left(\beta \mu_{0}\right) \equiv \frac{\left(p_{i}-p_{c}\right)}{\left(1-p_{c}\right)}<1
$$

Equation (22) relates the model parameters $\beta$ and $\mu_{0}$ to $p_{c}$ and to $p_{i}$, thus to the concentration $X$ and also to factors related to the microstructure of the particles such size and shape, which are discussed in Sec. VI.

The parameter $\beta$ is analogous to that introduced in SIM and must be positive [see discussion below Eq. (16) in Sec. III A], then $\mu_{0}$ must be negative. Actually, $\mu_{0}$ provides the strain value at whose vicinity a change in regime (inflection) is observed, defining the useful dynamic range when using the composite as a sensor of pressure. For instance, according to Eq. $(21),\left(p(\varepsilon)-p_{c}\right)$ must increase with $|\varepsilon|$ from $\left(p_{i}\right.$ $\left.-p_{c}\right)$ up to a plateau equal to $\left(1-p_{c}\right)$ which is actually reached when $|\varepsilon| \gg\left|\mu_{0}\right|$. On the other hand, at low strains when $|\varepsilon| \ll\left|\mu_{0}\right|$, a scale-invariant expression is obtained by taking the limits to low strains of the sigmoid distribution in Eq. (21), which leads to an exponential dependence at that limit

$$
\begin{aligned}
|\varepsilon| & \ll\left|\mu_{0}\right| \Rightarrow p(\varepsilon)-p_{c} \cong\left(1-p_{c}\right) \exp \left(-\beta\left(\varepsilon-\mu_{0}\right)\right) \\
& =\left(1-p_{i}\right) \exp (-\beta \varepsilon)
\end{aligned}
$$

which is coincident with the SIM result Eq. (17). Thus, $\beta$ provides the rise of $p(\varepsilon)$ at low stresses.

The above expressions for the dependence of $p$ with $\varepsilon$ can be used to obtain $\Gamma_{\|}(\varepsilon)$ and then $\sigma / \sigma_{i}$ and $G / G_{i}$. For instance:

$$
\Gamma_{\|}(\varepsilon)=\beta t(\varepsilon)\left(1-\frac{p(\varepsilon)-p_{c}}{1-p_{c}}\right)=\frac{\beta t(\varepsilon)}{1+\exp \left(-\beta\left(\varepsilon-\mu_{0}\right)\right)},
$$

$$
\begin{aligned}
\frac{G(\varepsilon)}{G_{i}}= & \exp (-\varepsilon) \frac{\left(1-p_{c}\right)^{t(\varepsilon)}}{\left(p_{i}-p_{c}\right)^{t_{i}}} \frac{1}{\left(\exp \left(\beta\left(\varepsilon-\mu_{0}\right)+1\right)\right.} \\
& \times \exp \left(\int_{\varepsilon}^{0}\left(\frac{d t}{d \varepsilon}\right) \ln \left(\frac{\left(1-p_{c}\right)}{\exp \left(\beta\left(\varepsilon-\mu_{0}\right)+1\right.}\right) d \varepsilon\right) .
\end{aligned}
$$

These expressions constitute the predictions of PSM for the experimentally accessible properties, $G$ and $\Gamma_{\|}$. They include $t(\varepsilon)$ which is discussed in the next section.

\section{MODELING THE CRITICAL EXPONENT FUNCTION, $t(\varepsilon)$}

As mentioned in the previous sections, nonuniversal behavior for $t$ has been experimentally reported. Moreover, in the limit of low particle concentration (the weight fraction $X$ close to a critical value $X_{c}$ ) and low strains, where tunneling can be the predominant process for conduction, the TPM predicts nonuniversal behavior for $t$. Actually, the TPM predicts that in systems where a mean-distance between neighbor particles $\langle r\rangle$ can be defined (which is not an obvious topics in concentrated systems or when large agglomerates of particles are present) then $t$ must be a linear function of $\langle r\rangle$ in those cases where departure from the SPT $(\mathrm{t}=2)$ are proposed or expected. This linear dependence is obtained by using the Kogut-Straley relationship ${ }^{17,48}$ in combination with the tunneling probability for the passage of an electron from a particle to another, which is an essential constituent of the TPM. In addition, Johner et al. ${ }^{17}$ reasonably proposed a direct proportionality between $\langle r\rangle$ and $\varepsilon$ in the range of very low strains considered by those authors, to finally obtain: $t(\varepsilon)=\nu+\mathrm{K}\langle\mathrm{r}\rangle=\nu+\mathrm{K}^{\prime} \varepsilon$, where $\nu$ is the correlation length exponent ( $\nu \approx 0.88$ for $3 \mathrm{D}$ materials) and $\mathrm{K}, \mathrm{K}^{\prime}$ are constant for the cases where $t>2$. The application of this model to a range of concentrated particles and moderated strains is not straightforward, because the concept of average distance between particles becomes vague and because physic contact between particles instead of tunneling is the main criterion for conduction. Nevertheless, it seems reasonable that one possible extension of the model can be made by exchanging the average distance between particles by the inverse of the particle volume fraction, $\phi^{-1}$, which is proportional to the length $L(\varepsilon)$ in the systems considered here where the area $A$ is kept constant. Then, as $L(\varepsilon)$ follows and exponential dependence with $\varepsilon$ [Eq. (3)], an exponential dependence between $t$ and $\varepsilon$ is deduced, although the boundary condition Eq. (9e) must be also considered. In conclusion, one reasonable extension of the model presented by Johner et al. in Ref. 17 is given by Eq. (26) which reduces to the expression developed in Ref. 17 in the limit of low strains and simultaneously satisfy the boundary condition [Eq. (9e)] is

$$
\begin{aligned}
& t(\varepsilon)=\nu+\left(t_{i}-\nu\right) \exp (\varepsilon) \text { if } t_{i}>2, \\
& t(\varepsilon)=2 \quad \text { if } t_{i}=2,
\end{aligned}
$$

where $\nu$ is the correlation length exponent, $\nu \approx 0.88$ for $3 \mathrm{D}$ materials [if the area $A$ is not kept constant, then $\varepsilon$ must be replaced by $\left(1-2 \nu_{P}\right) \varepsilon, \nu_{P}$ is the Poisson's modulus].

This formalism naturally leads to the still open debate about when nonuniversal effects can be experimentally detected, that is when $t_{i}>t_{0}=2$ under the condition that the concentration of conducting particles is large enough in order to obtain measurable values of conductance $\left(p_{i}>p_{c}\right)$. In the case of the pure tunneling mechanism, the condition for observing nonuniversal effects is that the average distance is 
not shorter than the characteristic tunneling distance otherwise pure contact applies, thus concentration must be kept below a given value or range. Therefore, at least when pure tunneling is the mechanism of conduction, the concentration of conducting particles used to load the elastomer must be restricted to a defined window in order to observe nonuniversal effects. If that condition is satisfied, then $t_{i}>t_{0}$ is expected and further variations of $t$ with the strain rendering departures from $t_{i}$ are expected. According to our hypothesis [Eq. (26)], an exponential variation in $t(\varepsilon)$ with $\varepsilon$ is predicted up to reaching the value $t=t_{0}=2$, and then a further compression does not modify the value of $t$. On the other hand, if the initial concentration does not range into the concentration window, then no dependence of $t$ with $\varepsilon$ is expected at all and the universal behavior $t=t_{0}=2$ is predicted in the whole range of strains.

In addition, there is a numerical aspect to consider when inspecting nonuniversal behavior, given by the fact that even when regarding a very broad range of strains the largest variations in real experiments are usually about $30 \%(|\varepsilon|$ $\leq 0.3)$ and therefore the deviation of $t(\varepsilon)$ from $t_{i}$ predicted [for example by Eq. (26)] should be in a limited range between 10 and $20 \%$ almost, depending on the specific value of $t_{i}$.

Summarizing, in practical terms there are only two possibilities for fitting experimental data: (i) universality: $t(\varepsilon)$ $=t_{i}=t_{0}=2$ for all $\varepsilon \leq 0$, or (ii) nonuniversality, where the exponential dependence is proposed: $t(\varepsilon)=\nu+\left(t_{i}-\nu\right) \exp (\varepsilon)$ with $\nu=0.88, \varepsilon \leq 0$ being $t_{i}$ a fitting parameter. However, the restrictive conditions discussed above represent a strong constraint for the experimental observation of the eventual dependence of $t$ with $\varepsilon$.

Finally, it is worth to note that PSM provides a simple expression for the case of universal behavior $\left[t(\varepsilon)=t_{i}=t_{0}\right.$ $=2]$

$$
\begin{aligned}
\frac{G(\varepsilon)}{G i} & =e^{-\varepsilon}\left(\frac{1+e^{\beta \mu_{0}}}{e^{\beta \varepsilon}+e^{\beta \mu_{0}}}\right)^{2} \quad(\mathrm{PSM}+\text { Universality }), \\
\Gamma_{\|}(\varepsilon) & =\frac{2 \beta}{1+\exp \left[-\beta\left(\varepsilon-\mu_{0}\right)\right]} \quad(\mathrm{PSM}+\text { Universality }),
\end{aligned}
$$

with only two fitting parameters, $\beta$ and $\mu_{0}$.

\section{SIMULATIONS USING PSM}

Expression (25) is used altogether with those obtained for the dependence of $t$ with $\varepsilon$ [Eq. (26)] for obtaining the dependence of $G(\varepsilon)$. Simulated dependences calculated for $t(\varepsilon), p(\varepsilon)-p_{\mathrm{c}}$, and $\Gamma_{\|}(\varepsilon)$ are presented in Fig. 1. The different values of $t_{i}, \beta$, and $\mu_{o}$ used in these simulations were estimated from the experimental fits of $G(\varepsilon)$ (see Sec. VI).

The stress range for variation in $p(\varepsilon)-p_{c}$ is determined by $\beta$ and $\mu_{0}$ as illustrated in Figs. 1(a) and 1(b), showing the sigmoid behavior, Eq. (21). Figure 1(c) shows the predicted dependence of $t(\varepsilon)$ according to Eq. (26). The departure of $t(\varepsilon)$ from $t_{i}$ is no larger than $13 \%$, even for largest values of $t_{i}$ in the range of stresses considered. The main consequence is that $G_{2}(\varepsilon)$ in Eq. (12) is numerically similar to $(p(\varepsilon)$ $\left.-p_{c} / p_{i}-p_{c}\right)^{t i}$, which is independent of $p_{c}$ in PSM. In fact, we have observed none or very poor influence of the $p_{c}$ value in simulations of $G_{2}$ and $G / G_{i}$ (Fig. 2) for $p_{c}$ between 0.1 and 0.3 .

The predicted dependence of $\Gamma_{\|}$with $\varepsilon$ according to Eq. (24) is shown in Fig. 1(d) for different values of $t_{i}$. The sigmoid term controls the behavior (in agreement with the comment of the previous paragraph), with inflection determined by $\mu_{0} . \Gamma_{\|}$reaches a plateau at the limit $\varepsilon \rightarrow 0$ and the limit value is proportional to $t_{i}$.

The plots of $\Gamma_{\|}$versus $G / G_{i}$ shown in Fig. 2 have similar shape than those reported by Johner et al. ${ }^{17}$ using effectivemedium approximation (EMA) simulations for a square lattice network of channels with resistive links between particles. That is, PSM provides results in qualitative agreement with EMA. Simulations of $G_{2}, G_{3}, G / G_{i}$, and $R / R_{i}$ as functions of $\varepsilon$ are shown in Fig. 3.

All the experimental characteristics associated to the dependence of $G$ with $\varepsilon$ are found in the simulations. The main contribution to $G / G_{i}$ is provided by $G_{2}$, which displays the largest variations with $\varepsilon$. The contribution of $G_{3}$ is not numerically relevant in the considered stress range. The dependence with $t_{i}$ is very strong; particularly, very large changes of $G_{2}$ and $G / G_{i}$ are found when going from $t_{i}=3$ to $t_{i}=4$.

Figure 3(d) shows the variation in $R / R_{i}(R=1 / G)$ with $P / E(=-\varepsilon)$, resembling one of the more frequent manners of reporting the response of pressure sensors ( $R$ versus $P$ plots) and in qualitative agreement with previous works. ${ }^{1,35}$

The expressions obtained for $G / G_{i}$ are used in the next section to fit experimental data obtained in our laboratory.

\section{EXPERIMENTS}

\section{A. Films and sensors preparation}

The composite is an elastic matrix of polydimethylsiloxane (PDMS) containing a macroscopically homogeneous dispersion of conducting graphite particles. Carbon graphite (SAO-Argentina) was used with $95 \%$ w/w graphite purity provided by the manufacturer and no impurities were detected by $\mathrm{x}$-ray dispersive analysis.

PDMS was from Dow-Corning (Sylgard 184). The curing agent and base were mixed in proportions of 1:10 or 2:10 at room temperature. These samples were loaded with carbon particles (the amounts of carbon and PDMS were weighted during mixing on an analytical balance), homogenized, and placed at room temperature in a vacuum stove for about two hours assuring the complete absence of any air bubble. This mixture, still fluid, was placed on the bottom metallic surface of the prepared sensor which was in turn placed inside of a delrin cylinder to avoid leakage of the fluid composite and then cured at $50{ }^{\circ} \mathrm{C}$ during $24 \mathrm{~h}$. After curing, films of about $1 \mathrm{~mm}$ thickness were obtained, whose top surfaces were then polished. The resulting films were observed by scanning electron microscopy (SEM, Fig. 4), where the high density of carbon particles (with average particle size about $1 \mu \mathrm{m}$ ) is observed together with a porous structure.

Samples with different amount of graphite particles were prepared in the range $10 \%-70 \%$ weight percentage (w/w \%). Appreciable conduction was only obtained for samples with 

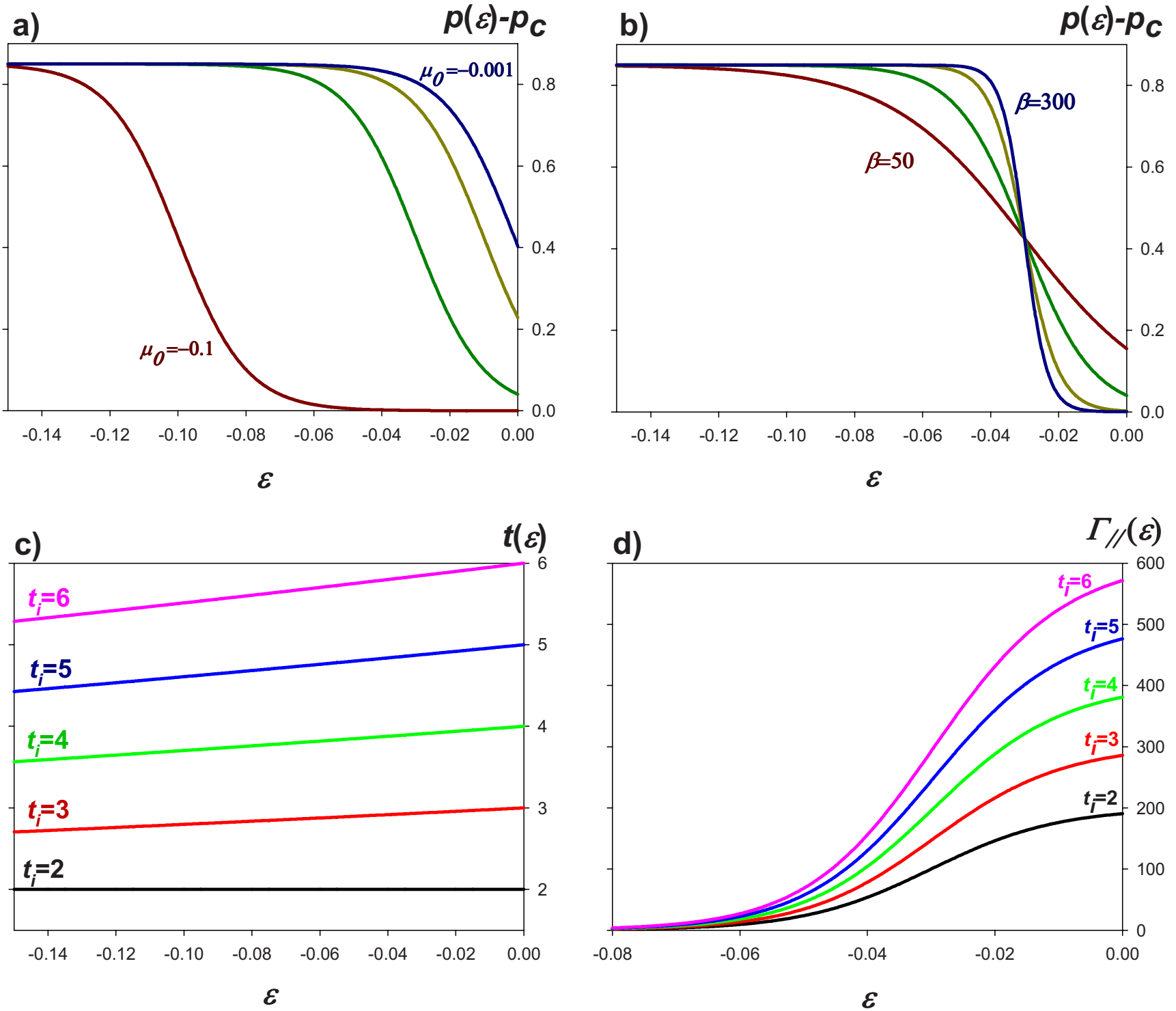

FIG. 1. (Color online) Simulations according to PSM. (a) $p(\varepsilon)-p_{c}$ for fixed $\beta(=100)$ and $p_{c}(=0.15)$ varying $\mu_{0}(=-0.1,-0.03,-0.01$, and -0.001 from left to right). (b) $p(\varepsilon)-p_{c}$ for fixed $\mu_{0}(=-0.03)$ and $p_{c}(=0.15)$ varying $\beta(=50,100,200,300)$. (c) $t(\varepsilon)$ [Eq. (15)] varying $t_{i}(\nu=0.88)$. (d) $\Gamma_{\|}(\varepsilon)$ [Eq. (24)] for fixed $\beta(=100)$ and $\mu_{0}(=-0.03)$.

weight percentage higher than $30 \%$. The ratio of densities between the prepared samples and the graphite $\left(2 \mathrm{~g} / \mathrm{cm}^{3}\right)$ was estimated about $0.5-0.75$, thus a draft estimation of the critical volume fraction of the conducting particles gives values between 0.15 and 0.22 .

\section{B. Elastic response}

Composites films for texture analysis were prepared by the procedure described in Sec. VI A. The dimensions of the films were between 1.5 and $2.0 \mathrm{~cm}$ diameter and between 0.8 and $1.5 \mathrm{~mm}$ thickness. The texture profile analysis was performed at $25{ }^{\circ} \mathrm{C}$ by means of a Stable Microsystems TAXT2i Texture Analyzer using a cylindrical probe with plane contact surface (P/36R $36 \mathrm{~mm}$ diameter). The films were compressed using that probe in the range between $8 \%$ to $30 \%$ of the initial thickness $(|\varepsilon|$ between $0.08-0.30)$ at a constant compression speed of $0.1 \mathrm{~mm} / \mathrm{s}$. Hence, the texture analyzer was used in this work to measure the force required to keep a constant compression speed when the probe uniaxi- ally compresses the material. The force is recorded at real time and observed on the screen of a dedicated computer and the primary information is obtained as force versus elapsed time plots [Fig. 5(a)]. The relevant elastic properties of the samples are recovered from those plots, particularly the value of $E$ which is necessary for applying the models of electrical transport. The initial thickness of the sample, $L_{i}$, is calculated by the software after the calibration procedure of the equipment. The experiments were performed in duplicate or triplicate. The texture analyzer was previously used for food textural studies and characterization by some of the co-authors. $^{49-52}$

Figure 5(a) shows the increase in pressure, $P$, on the film as response to the external compression at constant speed. The compression is continuously exerted up to a given maximum strain (a fixed percentage of $L_{i}$ ). The compression is released when reaching that maximum strain and then the force drops. The rise of the curves in Fig. 5(a) are determined by the particular value of $E$ of the sample. The varia- 


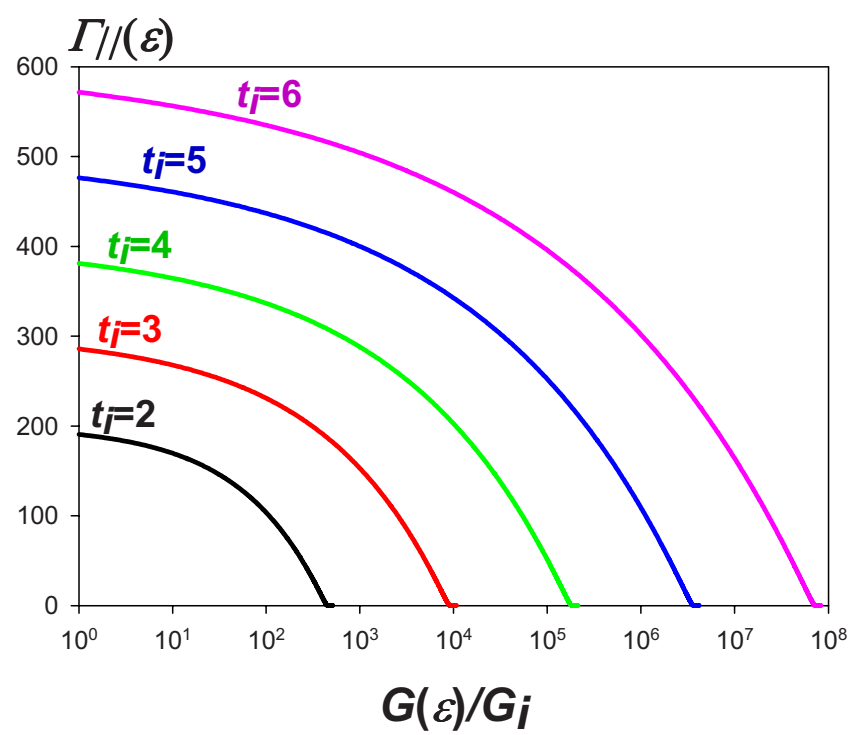

FIG. 2. (Color online) $\Gamma_{\|}$vs $(G / G i)$ for different values of $t_{i}$. $\beta=100, \mu_{0}$ $=-0.03$.

tion in $L$ with $P$ during the compression is calculated from the information recorded in those experiments. Curves of $L$ versus $P$ are shown in Fig. 5(b), which are fitted with excellent agreement by Eqs. (3) and (4), that is, the films follows the Young's law in the range of applied pressures. The values of $E$ were recovered from those fits. No statistical representative trend in the valued of $E$ was observed when changing the carbon concentration in the range 30\%-60\% w/w, which seems to be more influenced by differences of sample's preparation conditions, such as curing time and temperature. This issue is out of the slope of the present work. Thus, the value of $E$ was measured for each prepared films and the specific value was used when adjusting experimental curves of $G$ versus $\varepsilon$. The recovered values of $E$ were in the range of reported value for PDMS films, between 2.8 and $10 \mathrm{kgf} / \mathrm{cm}^{2} .39$

Other important aspect observed in Fig. 5(a) is the abrupt drop of the responses after eliminating the compression and also the absence of relaxation patterns (e.g., no oscillations), a clear indication that the response time of the sample to the presence/absence of applied pressure is in the order of $10^{-1} \mathrm{~s}$ or faster. This is also supported by the results of Fig. 5(d), which shows the responses for a sample that was compressed up to different maximum stress. The penetration distance was kept fixed during a given period of time (about $7 \mathrm{~min}$ ) after reaching the fixed compression. Figure 5(d) shows that the pressure remains almost fixed at the initial value without observing relaxation effects in that time scale.

In the experiments associated to Fig. 5(c), the force on the sample is not suppressed after reaching the maximum stress, but is gradually reduced (decompression) at the same rate that holds for compression. It is observed an apparent hysteresis for all samples, although the slope of $P$ versus elapsed time in Fig. 5(c) is the same for compressing and decompressing, indicating that the differences of the absolute values of $P$ between the curves is probably due to instrumental factors and not to the elastic behavior of the material. This is confirmed by the excellent reproducibility and coincidence of the $P$ versus time curves for all samples through several cycles of repetitions (at least after ten cycles). Moreover, the values of $E$ calculated in different cycles remain the same. All the prepared films behave as elastic materials with good partial recovery and each determination of $E$ was calculated with errors in the order of $0.05 \%$.

The observed variations in $E$ between 2.8 and $10 \mathrm{kgf} / \mathrm{cm}^{2}$ for the different samples are due to changes in polymer mixing (curing agent, initiator and carbon in the respective proportions), curing time and or curing temperature (typically $50{ }^{\circ} \mathrm{C}$ during $24 \mathrm{~h}$ ). It is not simple to control all these variables to obtain material of the exactly equal hardness. For this reason, the value of $E$ must be determined for each sample, as done in the present work.

\section{Electrical resistance measurements as function of the applied pressure}

For the balances experiments the films were placed between two metallic surfaces with full contact with the film. A fixed dc voltage, $V(9 \mathrm{~V})$, was applied between the surfaces and the film uniaxially compressed keeping $V=$ constant. Therefore, the trivial contribution, $G_{1}(\varepsilon)$, is always present although is numerically very close to 1 as discussed in Sec. II C. The samples were placed on a laboratory balance which allowed registering in real time the increase in force when applying a pressure on the top of the sample. A special setup was made which allows keeping area constant with good approximation. In fact, the device allows the possibility of exerting force by descending a metallic probe of fixed flat area $A$ on the sample from its top. The sample is confined in a cylindrical sample holder, with the same area $A$, coincident with the sample. The changes of electrical resistance, $R$, during compression were recorded with a data logger, simultaneously to the measurements of force (the values of $R$ can be dependent on the chosen value of $V$ ) and the electrical conductance, $G=R^{-1}$, calculated. The $I-V$ curves were measured for some samples at zero stress in the range $2-10 \mathrm{~V}$, observing a linear response in this range for the different samples tested (data not shown), which correspond to currents about 10-50 nA and resistances of about 0.5 to $1 \mathrm{M} \Omega$, depending on the sample and the applied stress. No electrical hysteresis with the applied voltage was observed in the $I$ - $V$ curves in the range $1-10 \mathrm{~V}$.

Some measurements of the electrical conductance were performed with the texture analyzer, where the top metallic surface compress the films at fixed speed $(0.1 \mathrm{~mm} / \mathrm{s})$ controlled by software. Electrical terminals were coupled to the probe in order to measure the electrical conductance simultaneously to the measurement of pressure during the compression. This methodology will be explored with more detail in future works.

\section{Comparison of PSM predictions with experimental results.}

Figure 6 shows experimental results of $G / G_{i}$ versus $\varepsilon$ for different samples with similar carbon concentration (about 55\% w/w). Comparison of the absolute values of 

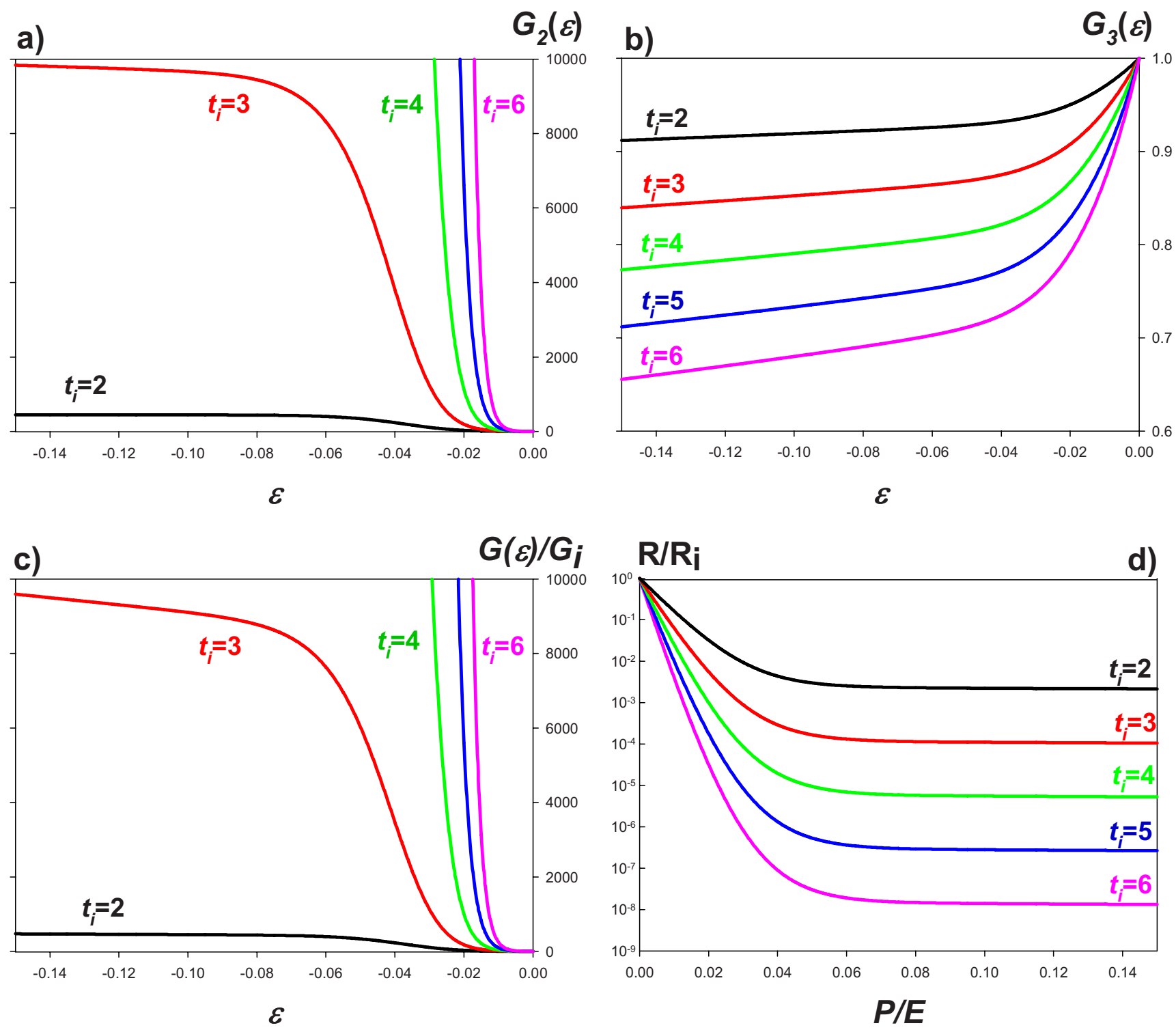

FIG. 3. (Color online) Simulations for fixed $p_{c}(=0.15), \beta(=100)$ and $\mu_{0}(=-0.03)$. (a) $G_{2}$, (b) $G$, (c) $G / G_{\mathrm{i}}$, (d) $R / R_{i},(R=1 / G)$ vs $P / E(=|\varepsilon|)$.

$G / G_{i}$ between different samples is not straightforward because are influenced by several experimental factors, like slight changes during preparation-curing, uncertainty in the determination of $G_{i}$ when currents are below $50 \mathrm{nA}$, and small variations in the percentage of present particles. However, all the samples have shown the behavior predicted by PSM. Figures 6(a)-6(d) correspond to compression in a balance, while Fig. 6(e) was obtained using the texture analyzer.

PSM plots [obtained using Eqs. (12), (21), and (26)] are superposed to the experimental points. These plots were calculated by arbitrary fixing the value of $t_{i}\left(t_{i}=2,3,4,5\right.$, and 6 although $t_{i}$ can take any real positive value) and then $\beta$ and $\mu_{0}$ were adjusted in order to minimize the deviation of the calculated plots with respect to the experimental points. Only the curves obtained for $t_{i}=2,3$ and 4 are shown for clearness. The best fits was obtained when using $t_{i}=2$ and 3 . Fits with $t_{i}=4$ were acceptable in three samples, but not for samples (a) and (b). Fits with $t_{i}>4$ were unacceptable in all samples. The values of $\beta$ and $\mu_{0}$ recovered for the case $t_{i}=2$ are shown in the insets for each sample. Values of $\beta$ between 55 and 300 and $\mu_{0}$ between $\left(-1 \times 10^{-3}\right)$ and $\left(-12.5 \times 10^{-3}\right)$ were obtained when considering all the samples and the acceptable fits.

When comparing the recovered values of $\beta$ and $\mu_{0}$ for the cases $t_{i}=2,3$, and 4 , we observed a slight tendency for $\beta$ to decrease, which is reasonable as the predicted characteristic stress is proportional to $1 /\left(1+\beta t_{i}\right)$ [see Eq. (26)]. The dependence of $\beta$ and $\mu_{0}$ with the carbon percentage deserves a very detailed experimental protocol, out of the present scope. These parameters are expected to vary with the chemical nature of the conducting particles and probably with its morphological aspects ${ }^{19}$ as discussed in Sec. VII.

It is worth to remark that the discussion of the results obtained when using pressure sensors must be done in terms of the strain, $\varepsilon$, and not on the stress $P$, actually. If the strain were not directly measured, the elastic parameters of the considered films should be determined. In the case of a uniaxial pressure parallel to the electrical current and with constant perpendicular area as considered here, the only parameter to be determined is $E$. If the discussion were presented in terms 

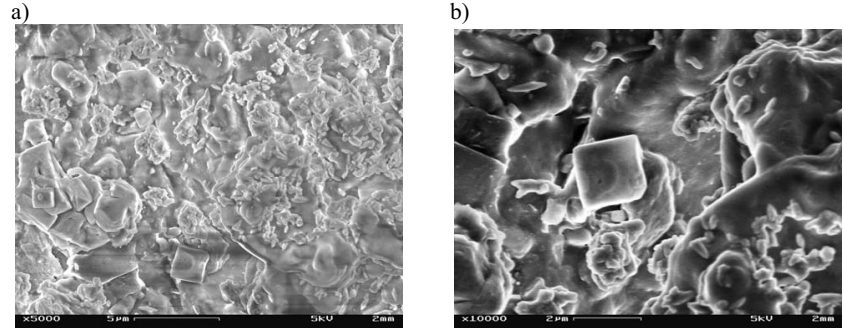

FIG. 4. SEM images of PDMS-graphite composites. (a) $\times 5000$; the bright areas correspond to graphite particles. (b) $\times 10000$; the darkest areas correspond to the PDMS matrix.

of $P$, then it must be taken into consideration that the PSM predicts a characteristic pressure in the variation in $G$ with $P$ of the order of $E / \beta t_{i}$, which is much lower than $E$. That is, while the change in $L$ with $P$ has a characteristic pressure equal to the Young's modulus $E$ [Eqs. (3) and (4)], the rise of $G(P)$ with $P$ at the initial pressures $\left(P \rightarrow 0, p>p_{c}\right)$ is predicted to be exponential with a much shorter characteristic pressure of the order of $E / \beta t_{i}$, according to the model developed here, indicating high sensitivity of pressure sensors at the lowest pressures, but also a shorter dynamic range of the sensor's response as function of $P$.

a)

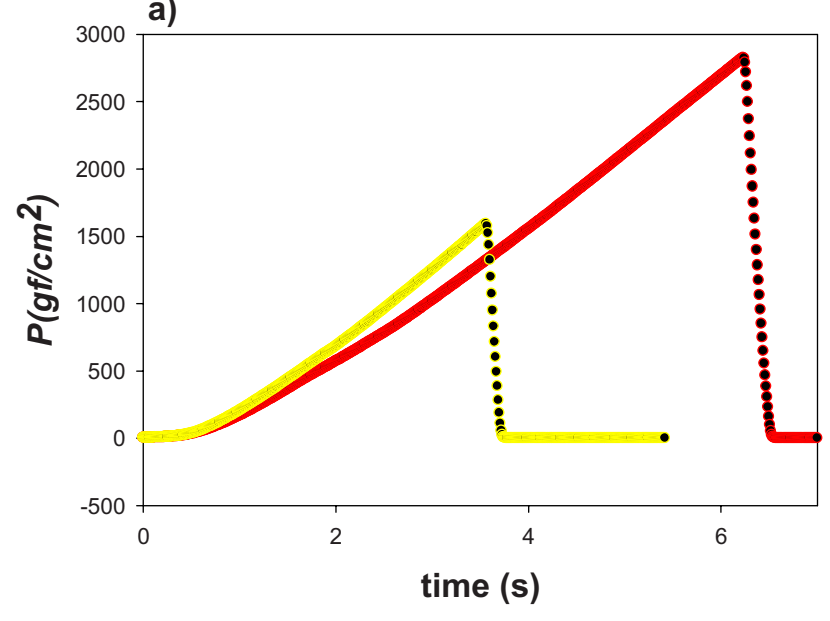

c)

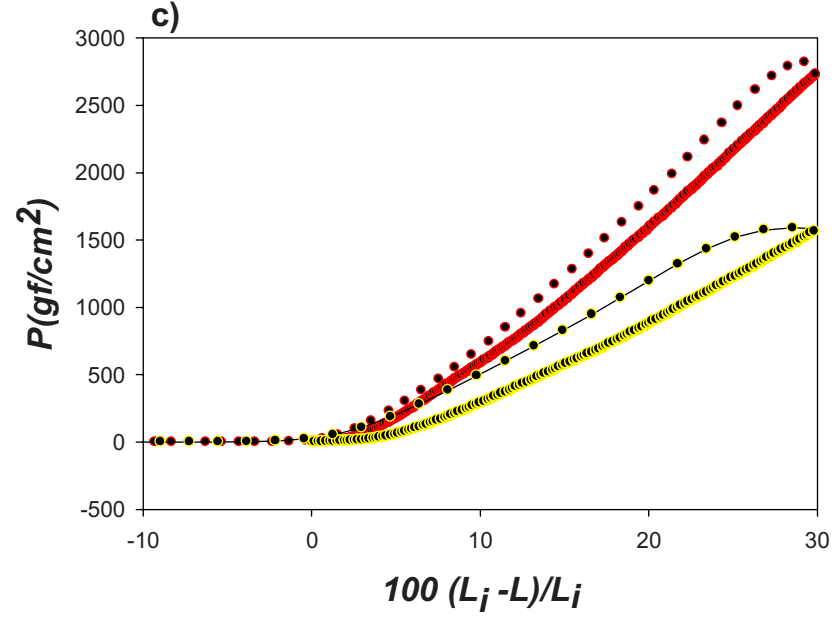

We present also a first result obtained with the texture analyzer [Fig. 6(e)]. In that experiment, the weight percentage of conducting particles was slightly lower than the critical value that we observed for conduction $(30 \% \mathrm{w} / \mathrm{w})$. Then, very low conduction was observed for that sample at $\varepsilon=0$ (resistance higher than $100 \mathrm{M} \Omega$ ). However, conduction appears at some critical pressure $\left[\mathrm{P}_{\mathrm{c}}\right.$ in Fig. 6(e)], about $3 \mathrm{kgf} / \mathrm{cm}^{2}$ for the considered sample when the sample is compressed with the texture analyzer. Thus, it seems that the percolation threshold is observed, although this must be taken as a preliminary result to be confirmed by further experiments. In fact, data points above $\mathrm{P}_{\mathrm{c}}$ seem to follow PSM while bellows that pressure the current remains almost constant or indistinguishable from the trivial variation.

The relatively large recovered values of $\beta$, in the order of 50-300, indicate that the main contribution to $G$ is provided by $G_{2}$. The trivial term $G_{1}$ is also shown to illustrate its very low contribution and change.

Data for silicone-graphite composites taken from Del Prete et $a l^{35}$ and Carmona et al. ${ }^{39}$ were also satisfactorily fitted by the PSM. The values of the Young's modulus $E$ were not reported by the authors and conductance data were

b)

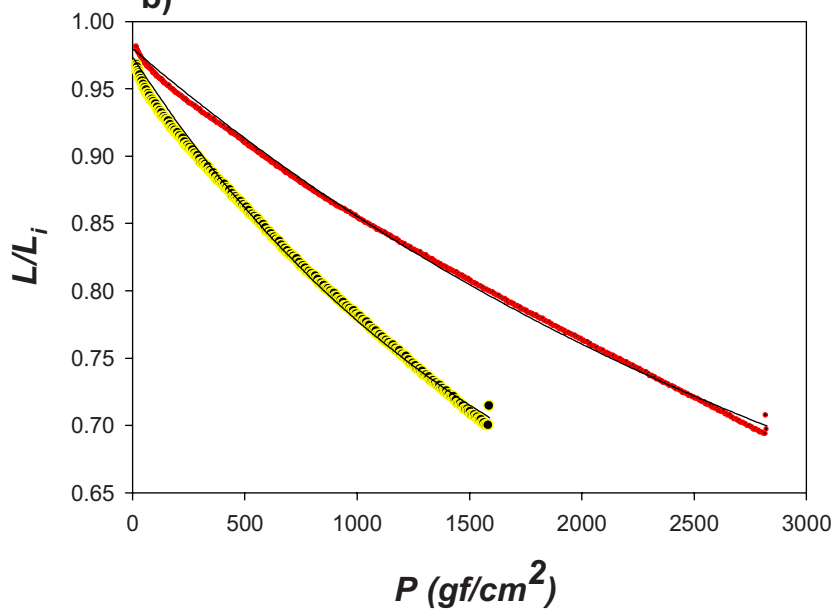

d)

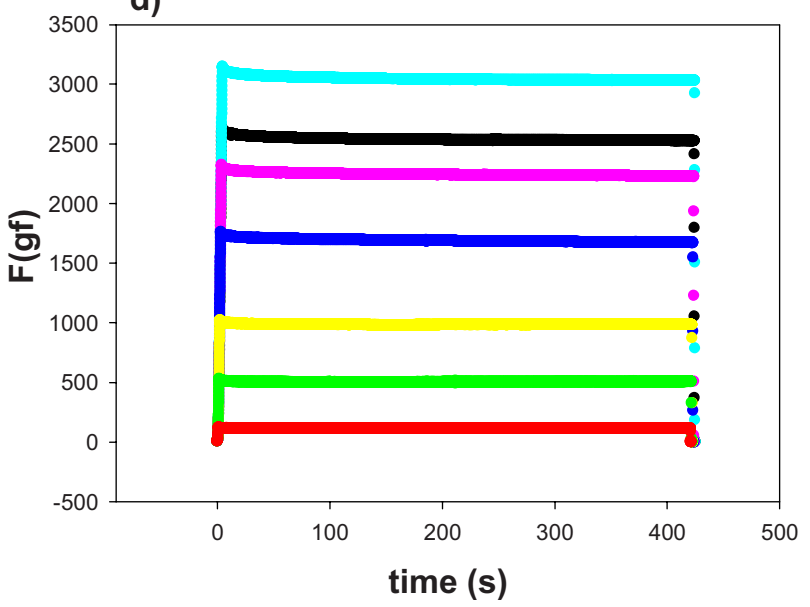

FIG. 5. (Color online) Texture analyzer results. Figure 5(a)-5(c) show curves obtained for samples of similar carbon concentration (55\% w/w) but obtained under different curing protocols. (a): samples of different initial thickness, $L_{i}$, are compressed up to $30 \%$ stress and then the probe is released. (b) $L / L_{i}$ vs $P$ plots. Data are well fitted by Eq. (3) for $L / L_{i}<0.975$. (d) Samples are compressed up to $30 \%$ and then decompressed at constant speed. (d) Response force as function of compression time: a given sampled is compressed up to different maximum stresses and then compression distance is kept fixed during $425 \mathrm{~s}$. 

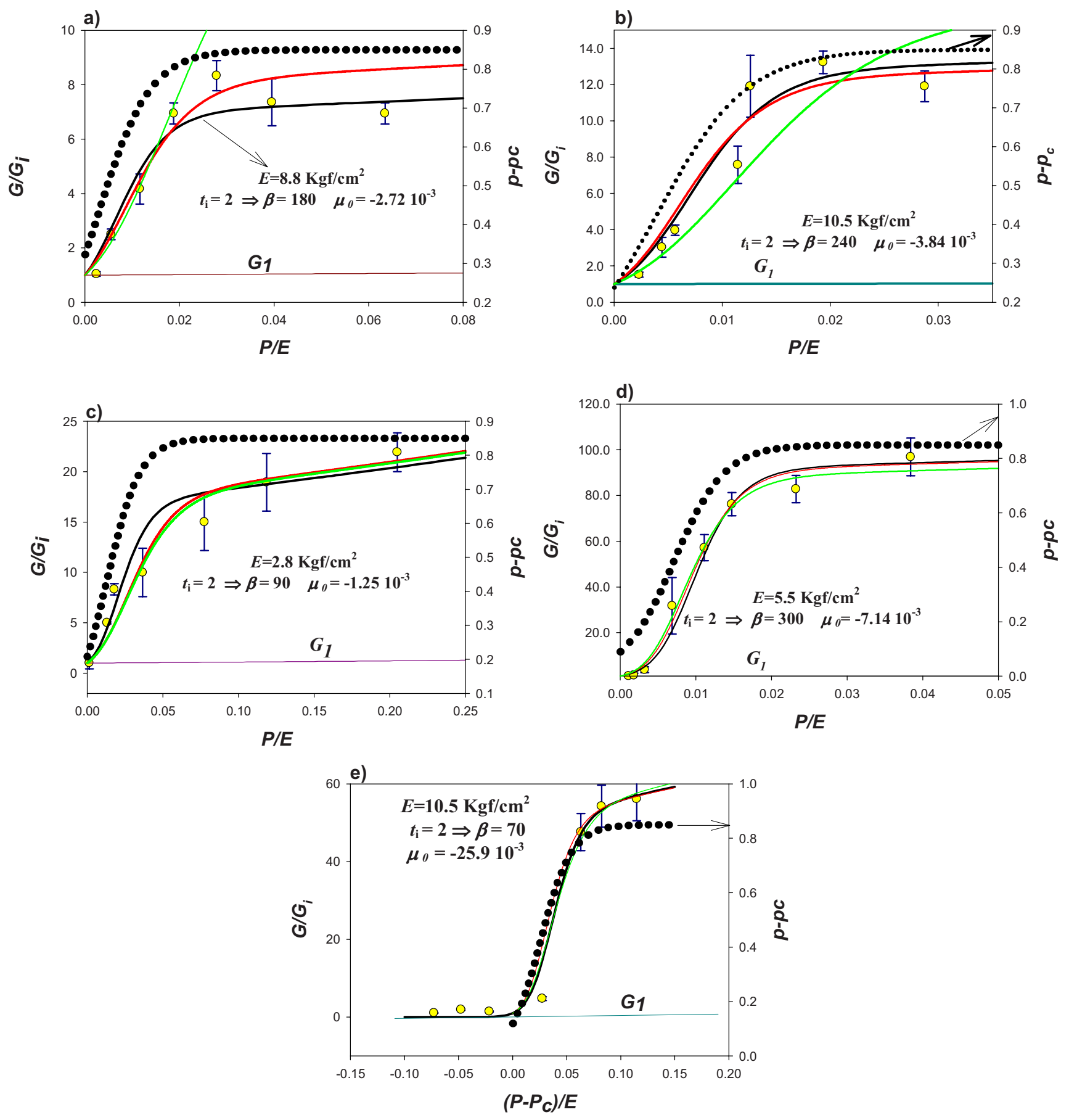

FIG. 6. (Color online) Experimental data (circle points with error bars) for $G / G_{i}$ vs $P / E(=|\varepsilon|)$ obtained for fifth samples with similar graphite concentration (55\% w/w). Fits with Eqs. (12), (21), and (26) are shown, obtained by fixing $t_{i}$ and optimizing $\beta$ and $\mu_{0}$. The values of $t_{i}$ were fixed to 2 (black lines), 3 (dark grey or red in the on-line version), 4 (light grey or green in the on-line version), 5 and 6 (not shown). No satisfactory fits were obtained for $t_{i}=5,6$. Very good fits were obtained with $t_{i}=2$ and 3 in all cases, while nonacceptable fits were obtained using $t_{i}=4$ for samples (a) and (b). The doted curves represent simulations of $\left(p-p_{c}\right)$ for the case $t_{i}=2$, calculated with Eq. (21) and the recovered $\beta$ and $\mu_{0}$ (and $p_{c}=0.15$ ). Figures 6(a) 6 (d) were obtained using the balance setup. Figure 6(e) was obtained with the texture analyzer $\left(\mathrm{P}_{\mathrm{c}}\right.$ represents here a critical pressure below what currents less than $1 \mathrm{nA}$ were obtained. The recovered values of $\beta$ and $\mu_{0}$ for $t_{i}=2$, and the measured $E$, are indicated in each case.

plotted as function of the applied pressure $P$, instead of the stress (thus we considered $E$ as a fitting parameter). Besides, data from those articles were reported as resistances in Ref. 35 and relative resistance changes in Ref. 39. Nevertheless, inflection points followed of a trend toward saturation can be observed when converting to conductance versus pressure plots, in agreement with the prediction of PSM. Indeed, we fitted the data reported by those authors by the PSM expres- sions presented here under universality conditions [fixing $t(\varepsilon)=2, \quad \mathrm{~d} t / \mathrm{d} \varepsilon=0]$ with excellent fits, recovering $E$ $\approx 10 \mathrm{kgf} / \mathrm{cm}^{2}$ for PDMS-carbon films in agreement with our own measurements of $E$ for silicone film using the texture analyzer $\left(E\right.$ between 2.8 and $\left.10 \mathrm{kgf} / \mathrm{cm}^{2}\right)$. For the data from del Prete et al., we recovered $\beta \sim 5 \times 10^{2}$ and $\mu_{0} \sim$ $-10^{-3}$, which are in agreement with the values recovered when fitting data of Fig. 6. 


\section{DISCUSSION AND FINAL REMARKS}

We present here an overview of the main results, possible criticism and discussion of some specific points. The main result is that the developed model, PSM, predicts correctly the experimental dependence of the electrical conductance $G$ as function of the strain $\varepsilon$ observed in experiments reported here and by other authors using graphite-elastomer composites. The main experimental features observed, a fast increase in $G$ under compression at the lowest applied pressures, followed by inflection of the response and finally saturation at moderated strains, are predicted by the PSM. These features were experimentally observed for example by Carmona et $a l .{ }^{39}$ who presented good fits of their data using a sigmoid dependence for $G$ as function of $P$, although deduced by assuming that the isothermal compressibility factor of the composite is proportional to the difference of isothermal compressibility factors of the polymer and the conducting particles and that this difference is a constant. In addition, no dependence of $t$ with $P$ was considered by those authors, but $t$ was assumed as a constant parameter during compression allowed to take any numerical value (higher than 2), which can be a good numerical approximation but is actually contradictory in the frame of elaborated theories like the TPM. Thus, the PSM presented here is more general by not assuming any special condition on the elastic properties of the composite and can be easily coupled to other models by allowing the possibility of incorporating universal or nonuniversal behaviors. The associated simulations of the piezoresistivity coefficient, $\Gamma_{\|}$, obtained with PSM are also in qualitative agreement with previous works using Monte Carlo simulations coupled to TPM.

PSM establishes that the critical behavior (scale invariance) still holds at low strains, but the percolation probability cannot increase indefinitely when strain increases, thus finally all the particles are connected to a percolation path. The mathematical expression for this physics is given in differential terms by $\mathrm{d}\left(p-p_{c}\right)=\beta\left(p-p_{c}\right)\left[1-\left(p-p_{c}\right) /\left(1-p_{c}\right)\right] \mathrm{d} \varepsilon$ (when keeping constant the $\mathrm{w} / \mathrm{w}$ fraction $X$ ) where $\beta$ is independent of $\varepsilon$. The factor $1 /\left(1-p_{c}\right)$ is a consequence of the boundary conditions defined in Eqs. (9a) and (9e), where $p_{c}$ is the critical value of $p$ for observing macroscopic conduction. The model converges at low strains to an scaleinvariant relationship between $p-p_{c}$ and the volume fraction occupied by the conducting particles.

The fitting parameters of the model are $p_{c}, \beta$, and $\mu_{0}$, see Eq. (21). However, the sensitivity of $G / G_{i}$ and $\Gamma_{\|}$to the specific value of $p_{c}$ is very low. Moreover, the expressions predicted by PSM are explicitly independent of $p_{c}$ in the case of universal behavior of $t$ as shown in Eqs. (27) and (28). Thus, the main parameters in PSM are $\beta$ and $\mu_{0}$.

The thermodynamics analogies presented by PSM, can be possibly elaborated further. For example, considering that $\beta \rightarrow-\left\{\partial\left[\ln \left(p-p_{c}\right) / \partial \varepsilon\right\}_{X}\right.$ when $\varepsilon \rightarrow 0$ and by noting that $\ln \left(p-p_{c}\right)$ must be related to the order of the system (lost of entropy) while $\varepsilon$ is associated to the elastic energy stored by compression in the composite, thus $1 / \beta$ should represent an effective temperature connecting entropic and energetic factors in the model.
Both $\beta$ and $\mu_{0}$ are, in principle, dependent on the weight fraction $X$. The relationship with $p_{i}[=p(\varepsilon=0)]$ is given by Eq. (22) which indicates that the product $\beta\left|\mu_{0}\right|$ must decrease with $p_{i}$, thus with $X$. The above discussion concerning the entropic-energetic factors suggest that $\beta$ must be very dependent on $X$, although to determine the details of the dependence of $\beta$ (and $\mu_{0}$ ) with $X$ through experiments is not an easy task.

The other factors that are expected to have a strong influence on $\beta$ and surely on $\mu_{0}$ are morphological factors related to the microstructure of the particles, like the size and aspect ratio. Although these concepts should be developed further, it seems that an increase in the aspect ratio of particles, from example when going from spheres to tubes, should lead to an increase in the contact-bonds between particles then to an increase in $p(\varepsilon)$ [associated to an increase in $\beta$, Fig. 1(b)] and, simultaneously, of the dynamic range of the composite [associated to $\mu_{0}$, Fig. 1(a)]. As mentioned in Sec. III B, $\mu_{0}$ determines the useful dynamic range when using the composite as a sensor of pressure, thus a strong influence of the aspect ratio of the conducting particles on $\mu_{0}$ is predicted, for example when using nanotubes as fillers.

A possible issue for further discussions arises in the fact that we did not assume tunneling or contact to be the main mechanism for conduction. The point of view assumed here is that in principle is difficult to determine which is the main mechanism, tunneling or physical contact, and that both should be present in a real sample. The variable $p$ represents always the probability of nonzero conduction between two neighbor particles.

Other issue of possible criticism is the validity of the assumptions and boundary conditions. The only assumption made for developing PSM is that $p$ can be created and destructed at moderated-high strains, a possibility already suggested previously in Ref. 21, but never developed as far as we know. On the other hand, the boundary conditions can perhaps be modified for specific experimental situations. However, the main features of the model should not be substantially modified.

Two specific topics of discussion concern with the values of $p_{c}$ and $t$. It was assumed here that $p_{c}$ is an intrinsic property of the material, independent of the strain. For instance, when different samples having different percentages of particles are considered (varying the w/w \%) without applying an external stress $(\varepsilon=0)$, then $p$ varies from sample to sample while $p_{c}$ is a constant. In this case, significant conductivity appears only in samples with $p>p_{c}$. Analogously, here we assumed that when a stress is applied on a fixed sample of a given amount of particles, $p$ changes but not $p_{c}$. Nevertheless, it is worth to remark that in the case of the universal model $\left(t=t_{i}=\right.$ constant $)$, the expressions obtained for $G / G_{i}$ in the PSM are independent of the value of $p_{c}$.

Concerning the critical exponent function $t$, the SPT predicts that $t$ is a universal constant $t_{0}$, but in more elaborated models $t$ can be not only different of $t_{0}$ but also a function of $\varepsilon, t=t(\varepsilon)$. Although this issue is not central to PSM, the possibility of nonuniversal behavior, including dependence of $t$ with $\varepsilon$ were incorporated through an exponential dependence, Eq. (16). This seems to be a reasonable extension to 
larger strains and concentrations of the concepts presented by Johner et al. ${ }^{17}$ However, we did not obtain concluding evidence for nonuniversal effects. For instance, very good fits of $G(\varepsilon) / G_{i}$ where obtained with $t=t_{0}=2$ (universal behavior) as well as with $t(\varepsilon)=\nu+\left(t_{i}-\nu\right) \exp (\varepsilon)$ with $t_{i}=3$. To decide between both possibilities requires not only more amount of experimental data but also under different conditions, such as precise determinations of the whole $I-V$ curve as function of the applied stress.

The combination of conductivity and elasticity measurements clearly show that the pattern in the $G$ versus $\varepsilon$ plots cannot be assigned to saturation of the elastic response, for example if $L(\varepsilon)$ had reached a plateau during compression. In fact, the variation in $L$ versus $\varepsilon$ is exponential (without observation of a constant term) or it is even lineal [see Fig. $5(\mathrm{~b})]$. That is, the saturation of $G$ with $\varepsilon$ is seen in a series of values of $\varepsilon$ where saturation is not observed in $L$.

In summary, the saturation cannot be assigned to saturation of the elastic response or to nonuniversality. The SIM model predicts no saturation, meaning that the scale invariance relations do not predict the observed behavior in the entire range of values of $\varepsilon$. In turn, the experimental results are explained by PSM. This model rationally predicts not only the plateau but also the initial exponential rise followed by inflexion, and converges to a SIM at low strains. PSM reproduces also previous simulations of $\Gamma_{\|}$versus $G$. Therefore, PSM predicts all the experimentally observed features.

\section{ACKNOWLEDGMENTS}

AP, FM, DLB, and RMN are research members of the Carrera del Investigador Científico (CONICET). Financial support was received from the University of Buenos Aires (UBACyT 2008-2010, Project No. X157), Agencia Nacional de Promoción Científica y Tecnológica (Grant No. PICT 2006-00568), and CONICET (Grant No. PIP 6382).

${ }^{1}$ N. Kchit and G. Bossis, J. Phys.: Condens. Matter 20, 204136 (2008).

${ }^{2}$ A. Sieber, P. Valdastri, K. Houston, C. Eder, O. Tonet, A. Menciassi, and P. Dario, Sens. Actuators, A 142, 19 (2008).

${ }^{3}$ Y.-M. Jia, F.-F. Wang, X.-Y. Zhao, H.-S. Luo, S. W. Or, and H. L. W. Chan, Compos. Sci. Technol. 68, 1440 (2008).

${ }^{4}$ H. Wang, H. Zhang, W. Zhao, W. Zhang, and G. Chen, Compos. Sci. Technol. 68, 238 (2008).

${ }^{5}$ M. Antal, G. Filipcsei, and M. Zrínyi, Compos. Sci. Technol. 67, 2884 (2007).

${ }^{6}$ B. Zhang, C. Xie, J. Hu, H. Wang, and Y. H. Gui, Compos. Sci. Technol. 66, 1558 (2006).

${ }^{7}$ Y. Song and Q. Zheng, Compos. Sci. Technol. 66, 907 (2006).

${ }^{8}$ Z. Varga, G. Filipcsei, and M. Zrinyi, Polymer 46, 7779 (2005).

${ }^{9}$ K. Arshak, E. Moore, L. Cavanagh, J. Harris, B. McConigly, C. Cunniffe, G. Lyons, and S. Clifford, Composites, Part A 36, 487 (2005).

${ }^{10}$ G. H. Kim, Compos. Sci. Technol. 65, 1728 (2005).

${ }^{11}$ M. Foygel, R. D. Morris, D. Anez, S. French, and V. L. Sobolev, Phys. Rev. B 71, 104201 (2005).

${ }^{12}$ T. Prasse, J.-Y. Cavaillé, and W. Bauhofer, Compos. Sci. Technol. 63, 1835 (2003).

${ }^{13}$ N. Johner, C.Grimaldi, T. Maeder, and P. Ryser, Phys. Rev. E, 79, 020104(R) (2009).
${ }^{14}$ I. Balberg, J. Phys. D: Appl. Phys. 42, 064003 (2009).

${ }^{15}$ W. Bauhofer and J. Z. Kovacs, Compos. Sci. Technol. 69, 1486 (2009).

${ }^{16}$ N. Johner, C. Grimaldi, I. Balberg, and P. Ryser, Phys. Rev. B 77, 174204 (2008).

${ }^{17}$ N. Johner, P. Ryser, C. Grimaldi, and I. Balberg, Phys. Rev. B 75, 104204 (2007).

${ }^{18}$ J. Z. Kovacs, B. S. Velagala, K. Schulte, and W. Bauhofer, Compos. Sci. Technol. 67, 922 (2007).

${ }^{19} \mathrm{~J}$. Li and J.-K. Kim, Compos. Sci. Technol. 67, 2114 (2007).

${ }^{20}$ C. Grimaldi and I. Balberg, Phys. Rev. Lett. 96, 066602 (2006).

${ }^{21}$ S. Vionnet-Menot, C. Grimaldi, T.Maeder, S. Strässler, and P. Ryser, Phys. Rev. B 71, 064201 (2005).

${ }^{22}$ C. A. Martin, J. K. W. Sandler, M. S. P. Shaffer, M.-K. Schwarz, W. Bauhofer, K. Schulte, and A. H. Windle, Compos. Sci. Technol. 64, 2309 (2004).

${ }^{23}$ D. Toker, D. Azulay, N. Shimoni, I. Balberg, and O. Millo, Phys. Rev. B 68, 041403(R) (2003).

${ }^{24}$ I. Balberg, Carbon 40, 139 (2002)

${ }^{25}$ G. E. Pike and C. H. Seager, Phys. Rev. B 10, 1421 (1974).

${ }^{26}$ V. Tomer and C. A. Randall, J. Appl. Phys. 104, 074106 (2008).

${ }^{27}$ G. Ambrosetti, N. Johner, C. Grimaldi, T. Maeder, P. Ryser, and A. Danani, J. Appl. Phys. 106, 016103 (2009).

${ }^{28}$ J. J. Hernández, M. C. García-Gutiérrez, A. Nogales, D. R. Rueda, M. Kwiatkowska, A. Szymczyk, Z. Roslaniec, A. Concheso, I. Guinea, and T. A. Ezquerra, Compos. Sci. Technol. 69, 1867 (2009).

${ }^{29} \mathrm{C}$. Li, E. T. Thostenson, and T.-W. Chou, Compos. Sci. Technol. 68, 1227 (2008).

${ }^{30}$ D. W. Hatchett and M. Josowicz, Chem. Rev. 108, 746 (2008).

${ }^{31}$ R. Zhang, M. Baxendale, and T. Peijs, Phys. Rev. B 76, 195433 (2007).

${ }^{32}$ Z.Wang, M.Lua, H-L.Li, and X.-Y. Guo, Mater. Chem. Phys., 100, 77 (2006).

${ }^{33}$ K. Yamaguchi, J. J. C. Busfield, and A. G. Thomas, J. Polym. Sci., Part B: Polym. Phys. 41, 2079 (2003).

${ }^{34}$ L. Flandin, A. Hiltner, and E. Baer, Polymer 42, 827 (2001).

${ }^{35}$ Z. Del Prete, L. Monteleone, and R. Steindler, Rev. Sci. Instrum. 72, 1548 (2001).

${ }^{36}$ M. Hussain, Y.-H. Choa, and K. Niihara, Composites, Part A 32, 1689 (2001).

${ }^{37}$ H. Krassow, F. Campabadal, and E. Lora-Tamayo, Sens. Actuators, A 82, $229(2000)$

${ }^{38}$ J. N. Aneli, G. E. Zaikov, and L. M. Khananashvili, J. Appl. Polym. Sci. 74, 601 (1999).

${ }^{39}$ F. Carmona, R. Canet, and P. Delhaes, J. Appl. Phys. 61, 2550 (1987).

${ }^{40}$ D. S. McLachlan, G. Sauti, and C. Chiteme, Phys. Rev. B 76, 014201 (2007).

${ }^{41}$ C. Chiteme, D. S. McLachlan, and G. Sauti, Phys. Rev. B 75, 094202 (2007).

${ }^{42}$ J. G. Meier, J. W. Mani, and M. Klüppel, Phys. Rev. B 75, 054202 (2007).

${ }^{43}$ G. R. Grimmett, Percolation (Springer, New York, 1989).

${ }^{44}$ A. G. Hunt, Percolation Theory for Flow in Porous Media, Lect. Notes Phys. (Springer, Berlin, 2005), Vol. 674.

${ }^{45}$ A. Trionfi, D. H. Wang, J. D. Jacobs, L.-S. Tan, R. A. Vaia, and J. W. P. Hsu, Phys. Rev. Lett. 102, 116601 (2009).

${ }^{46}$ M. Koehler, C. D. Canestraro, C. Schnitzler, M. M. Oliveira, A. J. G. Zarbin, L. S. Roman, and M. G. E. da Luz, EPL 79, 47011 (2007).

${ }^{47}$ R. García Molina, E. Louis, O. Pla, and F. Guinea, Phys. Rev. B 44, 9704 (1991).

${ }^{48}$ P. M. Kogut and J. P. Straley, J. Phys. C 12, 2151 (1979).

${ }^{49}$ J. Kobylañski, O. E. Pérez, and A. M. R. Pilosof, Thermochim. Acta 411, 81 (2004).

${ }^{50}$ O. Pérez, V. Wargon, and A. M. R. Pilosof, Food Hydrocolloids 20, 966 (2006).

${ }^{51}$ C. Capitani, O. Pérez, M. T. Bertoldo-Pacheco, and A. M. R. Pilosof, Food Hydrocolloids 21, 1344 (2007).

${ }^{52}$ O. Pérez and A. M. R. Pilosof, in Food Colloids, Biopolymers and Materials, edited by E. Dickinson and T. Van Vliet (The Royal Society of Chemistry, Cambridge, UK, 2003), p. 119. 